\title{
Benefit Incidence Analysis: Are Government Health Expenditures More Pro-Rich Than We Think?
}

\author{
by \\ Adam Wagstaffa , \\ ${ }^{a}$ Development Research Group, The World Bank, Washington DC, USA
}

Revised January 2011

\begin{abstract}
Authors of benefit-incidence analyses have to impute subsidies using assumptions about the relationship between unobserved subsidies "captured" by the household and what can be observed at the household and aggregate levels. This paper shows that one of the two assumptions used in BIA studies to date will necessarily produce a more pro-rich (or less pro-poor) picture of government health spending than the other, depending on whether utilization is more pro-rich or pro-poor than fees paid to public providers. Both assumptions have their disadvantages, and the paper suggests a couple of alternatives that explicitly link fees paid to the costliness of care. It shows that in the most likely case where fees are distributed in a more pro-rich fashion than utilization, the two traditional assumptions will produce less pro-rich distributions of subsidies than the two new alternatives. Also considered are three complications that arise in BIA studies, including factoring in social health insurance. The paper's theoretical results are illustrated with an empirical BIA for Vietnam.
\end{abstract}

Corresponding author and contact details: Adam Wagstaff, World Bank, 1818 H Street NW, Washington, D.C. 20433, USA. Tel. (202) 473-0566. Fax (202)-522 1153. Email: awagstaff@worldbank.org.

Keywords: benefit incidence analysis; government health spending; propoor health spending.

Acknowledgements: This paper grew out of a discussion with Aparnaa Somanathan, who also shared with me Vietnam's latest NHA data. I am grateful to Sarah Bales, Marcel Bilger, Davidson Gwatkin and two anonymous referees for very helpful comments on earlier versions of the paper. The findings, interpretations and conclusions expressed and any errors are entirely mine; the views expressed do not necessarily represent those of the World Bank, its Executive Directors, or the countries they represent. 


\section{Introduction}

Benefit-incidence analysis (BIA) has become an important tool for health sector analysts, the goal being to see whether, as policymakers apparently intend to be the case, government health expenditures (GHE) disproportionately benefit the poor (cf. e.g. O'Donnell et al. 2007) The major challenge facing the author of a BIA is that household surveys do not record the amount of government expenditure or subsidy that a household receives. Rather subsidies have to be imputed using assumptions that link what the analyst would like to observe at the household level-the subsidies "captured" by the household-to what can be observed at the household and aggregate levels. Remarkably, these assumptions - their nature, their plausibility and their implications-have largely gone unexplored up to now. Yet as this paper shows, they have implications for the estimated degree of pro-poorness (or pro-richness) of government spending.

The paper begins by examining the two alternative assumptions encountered in BIA studies to date: the constant unit subsidy assumption, and the constant unit cost assumption. It finds that one of these will necessarily produce a more pro-rich (or less pro-poor) picture of government health spending than the other, depending on whether utilization is more pro-rich or pro-poor than fees paid to public providers. It argues that both assumptions have their disadvantages, but that the constant unit cost assumption is especially problematic. Neither assumption uses the amount people pay in fees to the public sector as an indicator of the costliness of the care delivered. In reality, governments set fee schedules in part according to the costliness of the interventions delivered during an episode of care. The paper goes on to suggest a couple of further possible assumptions that link fees paid to the costliness of care received, one of which is very general and nests the other three as special cases. The paper 
derives expressions for the pro-poorness of subsidies under these assumptions, and concludes that in the most likely case where fees are distributed in a more pro-rich fashion than utilization, the two traditional assumptions will produce less pro-rich distributions of subsidies than the two new alternatives. For the most plausible assumptions, then, existing BIA studies are likely to make GHE look less pro-rich than it really is. This is confirmed in an empirical analysis of the distribution by income of Vietnam's GHE.

While the paper's main focus is on the core assumptions necessary to impute subsidies, three other issues receive attention, simply because at least one of the methods cannot be implemented without dealing with them. One concerns the decision about what to do when out-of-pocket payments to public providers recorded in the national health accounts (NHA) fall short of the grossed-up figure from the household survey. This turns out to be an issue in Vietnam, but is a common issue elsewhere too. In a BIA based on the constant unit cost assumption, the analyst has to decide which figure to use; a BIA that uses one of the other methods does not need to worry about it for reasons that are explained in the paper. In the case of Vietnam, the decision turns out to make an appreciable difference to the results. The second issue is what to do in the event that imputed subsidies turn out to be negative. This is likely to arise quite often when the constant unit cost assumption is used, and theoretically can arise with the two new assumptions under certain parameter values. Whether to truncate the imputed negative values at zero (the practice to date) or to keep them (and in effect allow for the possibility that for some units of care people may pay more than the unit cost) turns out to make an extremely large difference to the results-enough in the case of Vietnam to turn subsidies from being pro-rich to being pro-poor. The final issue dealt with in the paper is the role of health insurance in BIA. Studies to date in this genre have not accounted for the fact that in many countries (Vietnam included), public-sector providers receive budget allocations, fee 
income, and reimbursements from a government health insurer. These demand-side subsidies need to be added to the supply-side subsidies that a BIA traditionally allocates to households; otherwise, the analysis misses a key element of general government health expenditures. ${ }^{1}$ Factoring in health insurance reimbursements to providers complicates the analysis of the incidence of GHE. Under simplifying assumptions about the insurance arrangements in place, expressions can be derived for imputed subsidies, but tractable analytic expressions for inequality in subsidies cannot. Empirically, allowing for insurance reimbursements in Vietnam turns out to make very little difference to the degree of pro-poorness of GHE, reflecting the ushaped distribution of health insurance in that country and the shallow effective coverage there.

\section{The Role of Assumptions in Shaping the Estimated Incidence of Government Spending}

The aim of BIA is to estimate how government subsidies vary with income. Subsidies are not observed in the data, and therefore have to be imputed. This section sets out the various possible assumptions that have been made in the literature as well as some alternatives; it also shows how the assumptions affect the estimated degree of pro-poorness of government spending in the health sector.

Subsidies at the individual level are the difference between the costs incurred by publicsector providers and the fees paid by the user:

$$
S_{k i}=C_{k i}-F_{k i}=c_{k i} q_{k i}-f_{k i} q_{k i}=q_{k i}\left(c_{k i}-f_{k i}\right)=s_{k i} q_{k i},
$$

where $S_{k i}$ are the subsidies individual $i$ receives from subsector $k$ (e.g. hospital inpatient care), $C_{k i}$, are the costs incurred by providers in subsector $k$ in providing services to individual $i, F_{k i}$ are

\footnotetext{
${ }^{1}$ In the convention of NHA's, spending financed by mandatory social health insurance schemes is included as an element of general government health expenditure [cf. e.g. OECD, $2000 \# 6751$ p68].
} 
the fees paid by individual $i$ to providers in subsector $k, q_{k i}$ is the number of units of service of type $k$ consumed by individual $i$, and $c_{k i}, f_{k i}$ and $s_{k i}$ are the unit costs, fees and subsidies respectively for sector $k$ for individual $i$. At the individual level, only $F_{k i}$ and $q_{k i}$ are recorded in the household survey data. Aggregates of $S_{k i}$ and $C_{k i}$ are, of course, available. The goal of BIA is to estimate the distribution of the $S_{k i}$ by income.

\section{The constant unit subsidy assumption}

One assumption made in the literature (cf. e.g. Hammer et al. 1995) ${ }^{1}$ is that the unit subsidy, $s_{k i}$ is constant across all units of care of type $k$. We can compute this constant unit subsidy $\left(s_{k}\right)$ from the national health accounts (NHA). Aggregating across individuals, we get:

$$
\sum_{i} S_{k i}=s_{k} \sum_{i} q_{k i}
$$

and therefore

$$
s_{k}=\sum_{i} S_{k i} / \sum_{i} q_{k i}=S_{k} / Q_{k}
$$

i.e. the unit subsidy for subsector $k$ is equal to total subsidies for subsector $k\left(S_{k}\right)$ divided by total utilization for subsector $k\left(Q_{k}\right)$. From this we can compute individual i's subsidy for sector $k$ as follows:

$$
S_{k i}=\frac{S_{k}}{Q_{k}} q_{k i}
$$

The interest in BIA is how subsidies vary with household income. Inequalities by income in subsidies can be measured using the concentration index (Kakwani et al. 1997), a positive value of which indicates a pro-rich distribution, and a negative value of which indicates a propoor distribution. Since subsidies for subsector $k$ are assumed in this case to be proportional to 
utilization for subsector $k$, the concentration index of subsidies to subsector $k$ is equal to the concentration index for utilization:

(1) $\quad C I_{S_{k}}=C I_{q_{k}}$.

The concentration index for total subsidies is then a weighted average of the concentration indices for the various subsectors where the weight for subsector $k$ is equal to the sector's share in total subsidies, i.e.

$$
C I_{S}=\sum_{k}\left(S_{k} / S\right) C I_{q_{k}}
$$

Thus if all one wants to know is the concentration index for total subsidies, all one needs to know (under the unit subsidy assumption) is the share of total subsidies going to each subsector and the concentration indices for utilization for each subsector.

\section{The constant unit cost assumption}

An alternative assumption that is often made in BIA (cf. e.g. O'Donnell et al. 2007; O'Donnell et al. 2008) is that unit costs, $c_{k i}$, are constant across units of utilization. The constant unit cost, $c_{k}$, can also be got from the NHA. Aggregating across individuals, we get:

$$
c_{k} \sum_{i} q_{k i}=\sum_{i} S_{k i}+\sum_{i} F_{k i}
$$

and therefore:

$$
c_{k}=\left(S_{k}+F_{k}\right) / \sum_{i} q_{k i}
$$

i.e. the unit cost for subsector $k$ can be got by dividing the sum of aggregate subsidies and aggregate fee revenues by the aggregate number of units of utilization of subsector $k$. Hence we have 


$$
S_{k i}=\frac{S_{k}+F_{k}}{\sum_{i} q_{k i}} q_{k i}-F_{k i}
$$

A potential problem with the constant unit cost assumption is there is no assurance that the estimated $S_{k i}$ will always be positive. The response to this problem is often to replace negative estimates of $S_{k i}$ by zeroes (see e.g. O'Donnell et al. 2007 p96); this solution is discussed further below.

Under the constant unit cost assumption, the concentration index of subsidies to subsector $k, C I_{S_{k}}$, can be expressed in terms of the concentration indices for utilization, $C I_{q_{k}}$, and fees, $C I_{F_{k}}$. After some manipulation, we get:

$$
C I_{S_{k}}=\frac{C_{k}}{S_{k}} C I_{C_{k}}-\frac{F_{k}}{S_{k}} C I_{F_{k}}
$$

Given that costs are assumed to be proportional to utilization, we have

$$
C I_{C_{k}}=C I_{q_{k}}
$$

and therefore:

$$
C I_{S_{k}}=\frac{C_{k}}{S_{k}} C I_{q_{k}}-\frac{F_{k}}{S_{k}} C I_{F_{k}}
$$

From eqn (2), we see that the concentration index of subsidies is smaller the less concentrated utilization is among the better off. But it is also smaller the more concentrated user fees are among the better off. In other words, government spending looks less pro-rich if the better off pay higher fees for a given number of units of utilization. In fact, subsidies could turn out to be pro-poor if fees are sufficiently disproportionately concentrated among the better off, even if utilization is higher among the better off. It is also worth noting that if the concentration index for subsidies is computed from the concentration indices for utilization and fees using eqn (2), 
the result could be a concentration index that is smaller than -1 or larger than +1 (the standard range for the concentration index); this would happen when some individuals have negative subsidies.

\section{Assuming fees are proportional to unit costs}

Under the constant subsidy assumption, the subsidy received by person $i$ in subsector $\mathrm{k}$ is proportional to their utilization in subsector $k$. How much they pay out-of-pocket in user fees is irrelevant to the subsidy they receive. By contrast, in the constant unit cost approach, the more fees that someone pays for a given unit of utilization the smaller is the subsidy they receive.

A different approach would be to use the information on fees paid to learn something about the quantity and/or quality of the care received. If the better off pay higher fees than the less well off for the same utilization, it may be because they receive more services per unit of utilization; i.e. they are charged according to the services they receive. In many (perhaps most) countries, user fees are explicitly linked to the quantity of services rendered, rather than being flat rate for each unit of utilization (e.g. each outpatient visit). Fee schedules also often reflect the cost of the services rendered, e.g. more expensive drugs and tests have higher fees associated with them. When fees reflect the quantity and costs of services rendered, the better off may well be paying more in fees (if they do pay more) because they get more-or more expensive-tests or drugs for a given outpatient visit or inpatient admission.

So, an alternative to the two standard BIA assumptions would be to assume that costs vary across individuals according to the fees paid. As a first approximation, we could assume that unit fees and unit costs are proportionate to one another; this assumption is relaxed in the next subsection. Thus: 


$$
c_{k i}=\alpha_{k} f_{k i}
$$

where we expect $\alpha$ to be larger than 1 given that utilization is subsidized. We have:

$$
S_{k i}=\alpha_{k} f_{k i} q_{k i}-f_{k i} q_{k i}=\left(\alpha_{k}-1\right) f_{k i} q_{k i}
$$

The fraction $\left(\alpha_{k}-1\right)$ can be computed from aggregate data:

$$
\alpha_{k}-1=\frac{s_{k}}{F_{k}}
$$

Hence we have:

$$
S_{k i}=\frac{S_{k}}{F_{k}} f_{k i} q_{k i}=\frac{S_{k}}{F_{k}} F_{k i},
$$

so that total subsidies received by individual $i$ are proportional to the fees they pay, where the factor of proportionality is simply the ratio of subsidies to fees. Note that using this method, the estimated value of $S_{k i}$ is always nonnegative, assuming $\alpha_{k}>1$.

Under this alternative assumption, the concentration index for subsidies is simply equal to the concentration index for fees:

(3) $\quad C I_{S_{k}}=C I_{F_{k}}$.

Thus in contrast to the constant-cost assumption, under this proportionality assumption the more concentrated fees are among the better off, the greater is the pro-rich bias in the incidence of government spending.

\section{A more general assumption about the link between costs and fees}

The assumption of proportionality may be too strong. One could instead assume that unit costs are a linear function of fees: 


$$
c_{k i}=a_{k}+\alpha_{k} f_{k i}
$$

The intercept $a_{k}$ could be interpreted as the cost of providing a basic unit of care of type $k$; this cost is covered by the government out of general revenues. Fees are paid when the care is more costly than $a_{k}$. If $\alpha_{k}>1$, the fees paid do not fully cover the additional costs and subsidies are used to cover part of the costs of non-basic care. With the linearity assumption we have

$$
S_{k i}=\left(a_{k}+\alpha_{k} f_{k i}\right) q_{k i}-f_{k i} q_{k i}=a_{k} q_{k i}+\alpha_{k} f_{k i} q_{k i}-f_{k i} q_{k i}=a_{k} q_{k i}+\left(\alpha_{k}-1\right) F_{k i}
$$

In contrast to the constant-cost and proportionality assumptions, there are two parameters of interest here. However, they are linked by the aggregate relationship between subsidies and fees:

(5) $S_{k}=a_{k} q_{k}+\left(\alpha_{k}-1\right) F_{k}$.

Thus a higher value of $a$ means a lower value of $\alpha$, i.e. the larger the basic cost financed out of general revenues, the smaller the subsidy on non-basic care.

The concentration index for subsidies is now equal to:

$$
C I_{S_{k}}=\frac{a_{k} q_{k}}{S_{k}} C I_{q_{k}}+\frac{\left(\alpha_{k}-1\right) F_{k}}{S_{k}} C I_{F_{k}}
$$

i.e. it is a weighted sum of the concentration indices for utilization and fees, the weight on the former being larger the more general revenues are used to subsidize basic care. Using eqn (5) we can get an alternative expression for the concentration index for subsidies, namely:

$$
C I_{S_{k}}=\frac{a_{k} q_{k}}{S_{k}} C I_{q_{k}}+\frac{\left(S_{k}-a_{k} q_{k}\right)}{S_{k}} C I_{F_{k}} .
$$




\section{Comparing the various assumptions}

Figure 1 shows the different assumed relationships between unit costs and unit fees under the different assumptions, and indicates how unit subsidies (i.e. unit costs minus unit fees) change as fees change. Only when unit costs exceed unit fees is a subsidy involved, i.e. only when the individual is below the $45^{\circ}$ line in Figure 1 . The constant unit cost assumption assumes no relationship between unit costs and (unit) fees. This is the special case of the general assumption when $a_{k}=c_{k}$, and $\alpha_{k}=0$. Patients paying modest fees will receive a subsidy, i.e. will lie above the $45^{0}$ line. But those with sufficiently high fees will be recorded as having a negative subsidy; they will fall to the right of the $45^{\circ}$ line. The constant unit subsidy assumption, by contrast, assumes everyone-irrespective of the fees-gets the same unit subsidy. This is the special case where $\alpha_{k}=1$ and $a_{k}>0$. The gap between unit costs and unit fees stays the same whatever the fees paid-i.e. the constant-unit-subsidy line runs parallel to the $45^{\circ}$ line-and everyone receives a positive subsidy. Where costs are proportional to fees ( $a_{k}=0$ and $\left.\alpha_{k}>1\right)$, the gap between unit costs and unit fees subsidies increases with fees-the proportionality line gets further from the $45^{\circ}$ line as fees rise. Finally, in the general case with $a_{k}>0$ and $\alpha_{k} \neq 0$ the unit subsidy will increase with fees if $\alpha_{k}>1$ but will fall with fees if $\alpha_{k}<1$. In the latter case, unit subsidies will eventually become negative, though at a higher level of unit fee than in the constant cost case.

Table 1 collects the results for the subsidy concentration indices. In the constant unitsubsidy approach, we have $\alpha_{k}=1$ and $a_{k}=S_{k} / Q_{k}$, making eqn (6) collapse to eqn (1). In this case general revenues are concentrated on subsidizing the costs of basic care, and each dollar of cost incurred above the cost of basic cost is met by exactly one additional dollar of fee revenue. In the constant-cost approach, unit costs are constant and unrelated to fees, equivalent to setting 
$a_{k}=c_{k}$, and $\alpha_{k}=0$. Eqn (5) then collapses to eqn (2). The proportionality assumption, by contrast, amounts to assuming that there is no basic care not associated with the payment of fees $\left(a_{k}=0\right)$. Eqn (6') then reduces to eqn (3).

We can see the effect on the concentration index for subsidies of raising $a_{k}$ (and by implication lowering $\alpha_{k}$ ) by differentiating eqn ( $\left.6^{\prime}\right)$ with respect to $a_{k}$ to get:

$$
\frac{\partial C I_{S_{k}}}{\partial a_{k}}=\frac{q_{k}}{S_{k}} C I_{q_{k}}-\frac{q_{k}}{S_{k}} C I_{F_{k}}
$$

which is negative if

$$
C I_{F_{k}}>C I_{q_{k}}
$$

i.e. if fees are more pro-rich than utilization, the more emphasis there is on subsidizing basic care (i.e. the larger $a_{k}$ is), the more pro-poor (or less pro-rich) subsidies will be. If, by contrast, fees are less pro-rich than utilization, raising $a_{k}$ will increase the concentration index for subsidies.

Figure 2 shows the effect of raising $a_{k}$ for the (likely) case where fees are more pro-rich than utilization. (The ordering is reversed when fees are less pro-rich than utilization.) The bottom panel shows the link between $a$ and $\alpha$ from eqn (5): higher values of $a$ (the amount of basic coverage not requiring any fees) inevitably calls for lower values of $\alpha$ (the generosity of the per-unit subsidy on units of care where fees are levied). The top panel of Figure 2 shows how the concentration index for subsidies, $C I_{S_{k}}$, varies with a (or equivalently with $\alpha$ ). Moving from the proportionality assumption to the constant unit cost assumption is equivalent to raising $a_{k}$ from zero to the value of $a_{k}$ where $\alpha_{k}$ becomes zero. Intermediate values of $a_{k}$-above zero and below the point at which $\alpha_{k}$ becomes zero-will imply a distribution of subsidies that is 
less pro-rich than under the proportionality assumption but more pro-rich than under the constant-cost assumption.

\section{Insurance as a Complication}

There is a complication not considered above, or indeed in previous BIA studies, namely health insurance. It has been assumed from the start that providers cover their costs through subsidies or fees charged to patients. In many countries, however, providers (or at least hospitals) receive budget allocations and fee income but also income from public insurers; when someone is insured, all or part of the fees the person would have otherwise paid to the provider as an out-of-pocket payment are instead paid by the insurer to the provider.

How does this affect the analysis? We assume that insurance is mandatory and hence to be treated as part of general government expenditure on health (GGHE) in the NHA. The subsidy should therefore include not just government spending channeled through supply-side subsidies, but also reimbursements to providers by the social insurer. A more general formulation of the basic equation would therefore be

$$
S_{k i}\left(=G_{k i}+r_{k i} q_{k i}\right)=c_{k i} q_{k i}-f_{k i} q_{k i}=\left(c_{k i}-f_{k i}\right) q_{k i}=s_{k i} q_{k i},
$$

where $G_{k i}$ is government expenditure on health, $r_{k i}$ is the unit reimbursement for person $i$, and the subsidy to the health sector is equal to GGHE, i.e. government health expenditure plus the insurer's reimbursements to providers.

The general case, as was seen in the previous section, nests the other assumptions as special cases, so it makes sense to start with the general case and ask how insurance complicates matters. Rather than assuming that costs are linearly related to fees recorded in the 
survey, it makes sense to assume they are linearly related to the sum of fees and reimbursements received by providers; we denote this combined figure by $\tilde{f}_{k i}$. Thus we have:

$$
c_{k i}=a_{k}+\alpha_{k} \tilde{f}_{k i}
$$

To keep things simple suppose that the insurance scheme can be approximated by a proportionate coinsurance rate $\varphi$. Thus:

$$
\tilde{f}_{k i}=\left\{\begin{array}{l}
f_{k i} \text { if uninsured } \\
f_{k i} / \varphi \text { if insured }
\end{array}\right.
$$

so if $\varphi=0.75$ we scale up recorded fees by a factor of 1.5 to get the fees the individual would have paid without insurance, or equivalently the fees plus reimbursements that the provider receives. (We can compute $\varphi$ as aggregate fees paid by the insured divided by the sum of aggregate fees paid by the insured and reimbursements to providers by insurers. In the empirical illustration below we allow $\varphi$ to vary by type of service.) The subsidies for person $i$ are now equal to:

$$
S_{k i}=\left\{\begin{array}{c}
\left(a_{k}+\alpha_{k} f_{k i}\right) q_{k i}-f_{k i} q_{k i}=a_{k} q_{k i}+\left(\alpha_{k}-1\right) F_{k i} \text { if uninsured } \\
\left(a_{k}+\left(\alpha_{k} / \varphi\right) f_{k i}\right) q_{k i}-f_{k i} q_{k i}=a_{k} q_{k i}+\left(\left(\alpha_{k} / \varphi\right)-1\right) F_{k i} \text { if insured }
\end{array}\right.
$$

so that for given values of $a_{k} q_{k}, \alpha_{k}$ and recorded fees $F_{k i}$, the insured person receives a larger subsidy, reflecting the fact that the social insurer is paying part of the cost of the care received. Aggregating across the uninsured and the insured, we obtain:

$$
S_{k}=a_{k} q_{k}+\left(\alpha_{k}-1\right) F_{k}^{N I}+\left(\left(\alpha_{k} / \varphi\right)-1\right) F_{k}^{I}
$$

where $F_{k}^{N I}$ and $F_{k}^{I}$ are respectively the total (recorded) fees paid by the uninsured and insured. If we know $\varphi$, and assuming we know the total fees paid by the uninsured and insured, we can 
solve the equation above for $a_{k}$ (or $\alpha_{k}$ ) for a given value of $\alpha_{k}$ (or $a_{k}$ ). So, as before, $a_{k}$ and $\alpha_{k}$ are linked to one another by the aggregate relationship between subsidies, costs and fees.

What about the special cases and the implied concentration index for subsidies? In the case where unit costs are assumed constant $\left(a_{k} \neq 0\right.$ and $\left.\alpha_{k}=0\right)$, the analysis of the preceding section goes through as before. In the other cases, however, things change.

Consider the case where unit subsidies are assumed constant. This assumption has some plausibility if nobody is covered by insurance. But if insurance results in the insured paying only a fraction of the fees paid by those without insurance, unit subsidies (equal to the difference between unit costs and unit fees) cannot logically be constant across the insured and uninsured. What we can plausibly assume is simply that $a_{k} \neq 0$ and $\alpha_{k}=1$. In eqn (8), subsidies emerge as being proportional to utilization for the uninsured, but not for the insured; for the latter group subsidies depend on utilization but also the fees they pay because for each dollar of fee paid the insurer has also made some reimbursement on behalf of the insured person, and this adds to the subsidy. This means that the concentration index cannot in this case be computed simply as the concentration index for utilization. Rather, subsidies need to be imputed at the individual level using eqn (8), and then a concentration index for the resultant imputed subsidy needs to be computed. The value of $a_{k}$ can be computed using eqn (9).

In the proportionality case $\left(a_{k}=0\right.$ and $\left.\alpha_{k} \neq 0\right)$, from eqn (8) we see that subsidies are still proportional to fees but the factor of proportionality varies between the insured and uninsured. This reflects the fact that for the same utilization, the insured pay (and record) less in fees than the uninsured. The concentration index for subsidies is no longer therefore equal to the concentration index for fees, and has to be computed from the imputed subsidies obtained using eqn (8) and the value of $a_{k}$ obtained from eqn (9). 


\section{Data}

The rest of the paper illustrates empirically the role of different assumptions in determining the pro-poorness of government health spending, using Vietnam as a case study. The incidence of government spending in Vietnam has been the subject of several studies (Prescott 1997; Wagstaff 2005; O'Donnell et al. 2007; O'Donnell et al. 2008), all of which show a pro-rich distribution of government health spending. This section describes the data used in the exercise. The results are presented in section $\mathrm{V}$.

\section{The basic data}

To obtain results for all four methods, we require household data on per capita income or consumption (to rank households) and on utilization of public facilities, as well as NHA data on subsidies; for all methods other than the constant unit-subsidy method, we also require household data on out-of-pocket payments associated with the use of public facilities.

The results below use household-level data from the 2006 Vietnam Household Living Standards Survey (VHLSS). According to the VHLSS, over half of out-of-pocket payments (which, according to Vietnam's $\mathrm{NHA}^{2}$, financed $60 \%$ of health spending in 2006) were payments to public providers, the rest being split fairly evenly between spending on drugs and payments to private providers (mostly private clinics rather than hospitals) (Lieberman and Wagstaff 2009 p6). The survey records outpatient visits over a period of a year to the principal government providers of outpatient care, namely commune health centers (CHCs), polyclinics, and government hospitals (district and provincial). For each visit, respondents are asked to record the out-of-pocket payments associated with the visit. The survey also records inpatient admissions to the main government provider of inpatient care, namely government hospitals. Respondents are also asked to record the out-of-pocket spending they incurred during the 
admission. The survey thus has good data on $q_{k}$ and $F_{k}$ for the four major government subsectors. The survey includes weights that allow the survey data on utilization and fee payments to be grossed up to the population level. It also has a comprehensive measure of household consumption that reflects market expenditures as well as home production and the use value of housing and other household assets. We express this on a per capita basis to rank households by their living standards.

The exercise also requires data on total government spending (i.e. subsidies) for each subsector, as well as total out-of-pocket payments to the subsectors. The latter could come from grossing up the household data. Alternatively, they could come from fee data in the government statistics. The two will not necessarily give the same result, and in Vietnam do not in fact do so. Table 3 presents the NHA figures on government spending and fee revenues, and the note to the table explains how the figures are arrived at. Inevitably, assumptions have to be made in deriving the figures, as the NHA headings do not correspond exactly with the utilization breakdowns permitted by the household survey. Under 'personal health services' (item HD 1.1) the NHA records spending on inpatient and outpatient treatment by households and government (at the central, provincial and district levels), as well as spending by households on medicines for self-medication. Under 'health prevention and public health' (item HD 1.2) the NHA records spending by households and government (at the commune level) on primary health care and school health care'. The assumption made in Table 3 is that the spending on inpatient and outpatient care recorded in HD 1.1 refers to spending at the hospital level (district and above), while the spending on 'primary health care and school health care' recorded under HD 1.2 covers spending incurred in CHCs and polyclinics, as well as schools. In Table 3 spending on school health is assumed to account for $25 \%$ of the total, and the rest is allocated between 
CHCs and polyclinics pro rata according to utilization volumes. Neither assumption is particularly satisfactory, but is the best that can be done with the available data.

\section{Complications associated with out-of-pocket spending data}

As already noted, data on out-of-pocket payments create two problems for a BIA based on the constant unit cost assumption. One is the possibility of negative imputed subsidies. The other is the possibility of an inconsistency between out-of-pocket payments recorded in the NHA and the implied grossed-up figure from the household data.

The out-of-pocket spending figure reported in the health ministry's NHA data in Table 3 is much less than the grossed-up figures from the household survey in Table 2 . The gap between the health ministry's out-of-pocket figures and the survey figures is particularly pronounced in the case of inpatient care, where the official figure captures only $19.6 \%$ of the spending recorded in the survey. This discrepancy has been noted in previous work (Knowles et al. 2005 p12), where it was suggested that informal payments may account for part of the discrepancy. Transport costs are another likely source of discrepancy, since respondents in the VHLSS are asked to include "payments for medical service and treatment", but also "other related costs (e.g. bonus for doctors, transport)". Unfortunately there is no way to exclude transport costs from the household data.

BIA studies (those for Vietnam have followed this practice) typically believe the aggregate figure reported in the health ministry's statistics and assume that the survey data are wrong, due to inaccurate recall, or because they include payments for things other than health care, such as transport. Researchers then scale down the fees reported in the household survey by the ratio of reported aggregate user fees as a fraction of the NHA aggregates, with the result that the scaled user fees in the survey gross up to the NHA figure. Then the $F_{k i}$ are scaled fees 
and $F_{k}$ is the fee revenue reported in the NHA. An alternative approach, and not apparently used to date, is to believe the household survey data, and to assume that the ministry data may omit payments made by households to public providers. Government providers may hide fee revenues from the ministry of health, or they may collect unofficial payments. Both constitute payments for resources used in delivering health care, and in that sense ought to be included. ${ }^{3}$ In this approach, aggregate fees would be obtained from the survey, and aggregate costs for subsector $k$ would be obtained by adding government spending on the subsector (i.e. subsidies to the subsector) to the grossed-up user fees from the survey data.

If the proportionality assumption is made, it makes no difference whether one uses the grossed-up out-of-pocket payment data from the survey or the NHA figure, since everyone's payments are scaled up or down by the same fraction and $C I_{F_{k}}$ is unaffected, cf. eqn (3). By contrast, if the constant-cost assumption is made, whether one uses the grossed-up household survey out-of-pocket spending total or the NHA figure does make a difference. Scaling up or down the reported fees leaves $C I_{q_{k}}, C I_{F_{k}}$ and $S_{k}$ unaffected in eqn (2), but $C_{k}$ and $F_{k}$ are both affected, $F_{k}$ proportionately more than $C_{k}$. In the present case, where the survey leads to a larger aggregate out-of-pocket spending figure than the NHA, the effect of scaling down the survey data to gross up to the NHA figure is to put more weight on $C I_{F_{k}}$ in eqn (2). Finally, if the more general assumption of a linear relationship between costs and fees is made, it makes no difference whether the survey fee payments are scaled up or down to the NHA figure, at least in the case where one holds constant $a_{k}$. In this case, $\alpha_{k}$ adjusts to hold $\left(\alpha_{k}-1\right) F_{k} / S_{k}$ in eqn (6) constant, since otherwise the cost constraint, eqn (5), is no longer satisfied. 


\section{Complications associated with insurance}

In Vietnam, with the exception of insurance for school-children, there is just one government insurer that provides cover (at the taxpayer's expense) to the poor (and other vulnerable groups), (at the employer's and employee's expense) to formal-sector workers, and (at the taxpayer's expense) to civil servants, war veterans and other "people of merit". The insurer reimburses hospitals according to the same scale that the hospital uses to charge uninsured patients.

Implementing the additional methodological twist of section III to factor in insurance requires some additional information. First, we need an estimate of the coinsurance rate $\varphi$. For commune-level services (commune health centers and polyclinics), the NHA records zero SHI reimbursements to providers. For these two providers, we therefore set $\varphi=0$. For each of outpatient visits at district hospital level and inpatient care at district level, we divide grossed-up fees paid by the insured (from the household data) by the sum of grossed-up fees paid by the insured and the amount reimbursed to providers by the insurer. We obtain values of $\varphi$ equal to $67 \%$ and $72 \%$ respectively. In other words, $70 \%$ of provider income from insured patients comes from out-of-pocket payments by the insured; the insurer only picks up $30 \%$.

\section{Results}

This section reports the results of the empirical illustration, beginning with the results based on section III where the complications introduced by insurance are ignored, and then moving to the results where insurance is factored in using the methods outlined in section III. 


\section{Basic results}

Table 4 shows the inequality in the incidence of health sector subsidies in Vietnam, according to the various methods outlined above, and assuming away the insurance problem. The figures for total subsidies in each case are simply weighted averages of the concentration indices for each category of care where the weight is the share of subsidies for that type of care in total subsidies.

The results in Table 4 are presented in ascending order of the subsidy concentration index, i.e. moving right to left in Figure 2 (given fees are more pro-rich than utilization in this case). The first results are when the constant unit cost assumption is made. In this case (and only in this case), we run into the two complicating issues: what to do about negative imputed subsidies; and whether to use grossed-up survey data on fees or NHA numbers. Decisions in both cases affect the results. Unit costs are dramatically smaller when the NHA data are used in the case of hospital care reflecting the lower aggregate fee figure when the NHA data are used. This results in less (negative) weight being put on the concentration index for $F_{k}$ in eqn (2); as a result, subsidies appear to be more pro-rich (or less pro-poor) when the NHA data are used. An even bigger influence on the results under the constant-cost assumption is whether or not negative subsidies are set to zero. Irrespective of whether the grossed-up survey data or the NHA data are used, the effect of setting negative subsidies to zero is to change subsidies from being pro-poor to being pro-rich. In other words, it is highly likely that had previous studies of

the incidence of government health spending in Vietnam not adopted the practice of setting negative imputed subsidies to zero, the conclusion would have been that government health spending in Vietnam disproportionately benefits the poor $!^{4}$ 
It is worrying that what is done with negative subsidies makes such a difference to the results. It casts some doubt on the constant cost assumption and the view implicit in it that fees in general-and especially very large ones causing negative subsidies-tell us nothing about the cost of the care being received. The constant cost assumption treats negative subsidies as a nuisance and sweeps them under the carpet. By contrast, the proportionality assumption and the more general assumption both explicitly assume that fees paid tell us something about the cost of care, and that particularly high fees indicate that the care received has been especially high-cost.

The second set of results in Table 4 assume that unit subsidies are constant. In effect, the government allocates all its subsidies on basic care and people pay the full cost of units of care above this basic level. This is the general case where $\alpha=1$ and therefore $a_{k}=S_{k} / q_{k}$. In this case, the concentration index for subsidies reduces to the concentration index for utilization. Given the results in section II, and given that in this application fees are more pro-rich than utilization, subsidies in this case turn out inevitably to be more pro-rich than they are under the constant-cost assumption-whatever is done under the latter with negative fees and whether in the latter one uses grossed-up survey or NHA data for aggregate fees.

The third set of results in Table 4 relate to the general assumption where unit costs and fees are linearly related but $\alpha \neq 1$. In Table $4 a$ is set in terms of a fixed monetary amount, subject to $\alpha \geq 1$ from the budget constraint (5), i.e. fees cannot exceed unit costs at the margin. In the absence of any estimates of the basic unit cost of each type of care, illustrative values $a$ were chosen. For $\mathrm{CHC}$ visits and polyclinic visits the value of $a$ was set as close to (the Vietnamese Dong equivalent of) \$ US 1.00 without $\alpha$ falling below 1 from eqn (5). For hospital outpatient visits, $a$ was set at the equivalent of $\$ 2.00$, and for inpatient visits $a$ was set at the equivalent of 
$\$ 10.00$ Under these assumptions, $\alpha$ is $100 \%$ for $\mathrm{CHC}$ and polyclinic care, but larger than $100 \%$ for the other two types of care, i.e. units of care costing in excess of the cost threshold are also subsidized. These assumptions, as expected given Figure 2, result in a more pro-rich distribution of subsidies than under the constant subsidy assumption.

The final case considered is the case where unit costs and unit fees are proportional to one another. In this case the concentration index for subsidies is simply the concentration index for fees, which is the same irrespective of whether the grossed-up survey data are used or the NHA data. Given the heavy weight on hospital care in government spending and the highly prorich distribution of fees for these types of care, the distribution of subsidies emerges as highly pro-rich under the proportionality assumption-far more than under the constant cost assumption let alone the constant cost assumption.

\section{Results taking into account insurance}

Table 5 shows the results obtained using the methods set out in section III that allow for health insurance coverage. As mentioned in section III, the constant unit cost assumption produces the same results whether or not insurance is allowed for. In Table 5 it emerges that in the case of Vietnam, factoring in insurance makes little difference to the estimated concentration indices for the other cases. This likely reflects two factors: the high effective coinsurance rate of around 70\%, and the u-shaped distribution of insurance coverage across the income distribution, reflecting in turn the high coverage rate of the poor under the Health Care Fund for the Poor program (Wagstaff 2010) and the relatively high coverage rates among formal-sector workers, government officials, and other groups who are compulsorily insured (cf. e.g. Lieberman and Wagstaff 2009). With a u-shaped insurance coverage distribution by income, the concentration index for insurance is virtually zero. Had insurance been heavily concentrated 
among the rich, and had the coinsurance rate been much higher, factoring in insurance would have made a much bigger difference to the estimated inequality in health sector subsidies, making GHE more pro-rich when social insurance is factored in.

\section{Conclusions}

The aim of this paper was to examine the implications in BIA of different assumptions about the relationship between unit costs and unit fees. A common approach is to assume constant unit costs. Another is the constant unit subsidy approach, where government revenues are focused on subsidizing a basic unit of care and costs above the basic unit are met dollar-fordollar by extra fees. Two other approaches explored in the paper are that unit costs are proportional to fees, and that unit costs are a linear function of fees with intercept $a$ and slope $\alpha$. The latter is very general, and subsumes all the other assumptions as special cases. All except the constant cost assumption allow for the fact that higher fees are likely to reflect a more costly type of care in a particular unit of utilization.

Section II of the paper shows that if fees are more pro-rich than utilization, the proportionality assumption yields the most pro-rich distribution of subsidies and the constantcost assumption the least pro-rich distribution. The constant unit subsidy assumption yields the second least pro-rich outcome, while the linear general case where $\alpha>1$ yields the third least pro-rich outcome. With the constant unit cost assumption, there is the complication that imputed subsidies could turn out to be negative, and it could make a difference whether one uses grossed-up out-of-pocket spending from the household data or NHA data on fees paid to public facilities. Section III of the paper then explored the complications to BIA when there is a public insurer that reimburses providers part of the cost of care. 
The rest of the paper explored the incidence of government health spending in Vietnam under the different assumptions set out in the paper. Previous studies have made the constant unit cost assumption. This, as expected-given that fees are more pro-rich than utilization in Vietnam - gives the least pro-rich subsidy distribution. This is the case whatever one does under the constant unit cost assumption about negative imputed subsidies and whether one uses NHA data on user fees to public facilities or grossed-up household survey data. In one case, in fact, the constant unit cost assumption produces a pro-poor distribution of subsidies. The other three assumptions produce more pro-rich subsidy distributions in the expected order, with the constant unit subsidy yielding the second least pro-rich, and the proportionality assumption producing the most pro-rich subsidy distribution. In the particular case of Vietnam, factoring in insurance coverage makes little difference to the estimated pro-poorness of government health spending. The results would be more sensitive to factoring in demand-side subsidies through insurance when social insurance coverage is highly unequally distributed between the rich and the poor, and when social insurance dramatically reduces the amount people pay out-of-pocket. This is the situation in many Latin American countries, where social insurance has typically not been factored into benefit-incidence studies.

There is no right or wrong assumption about the relationship between unit costs and unit fees. However, the constant unit cost assumption is arguably the least plausible of the four. It implies that higher fees do not translate into more costly care. This suggests an odd price schedule in the public sector, and begs the question of why people voluntarily pay higher fees if they do not get more costly care in return. The constant unit cost approach can, as already mentioned, produce negative imputed subsidies. This is unattractive, not least because imputed subsidies no longer gross up to the NHA subsidy figures. At the other extreme, the proportionality assumption is probably too strong, since most governments provide some basic 
care free of charge. The general linear assumption may be the most plausible, but it is hard to operationalize in the absence of knowledge about the parameters linking unit costs and unit fees. The constant unit subsidy assumption-which is the special case of the general linear assumption where $a$ is simply the unit subsidy and $\alpha=1-$ might be a reasonable simplification of the general linear case. It also has the great merit of being the least demanding assumption in terms of data: it does not require microdata on fees paid by households to different types of provider. But this is only true where there is no social health insurance reimbursement to worry about. Where the government channels subsidies through the demand-side through social health insurance, as is increasingly the case, these subsidies need to be factored in and even the constant-unit-subsidy approach requires data on fees paid to providers. 
Figure 1: Links between unit costs and unit fees under different assumptions

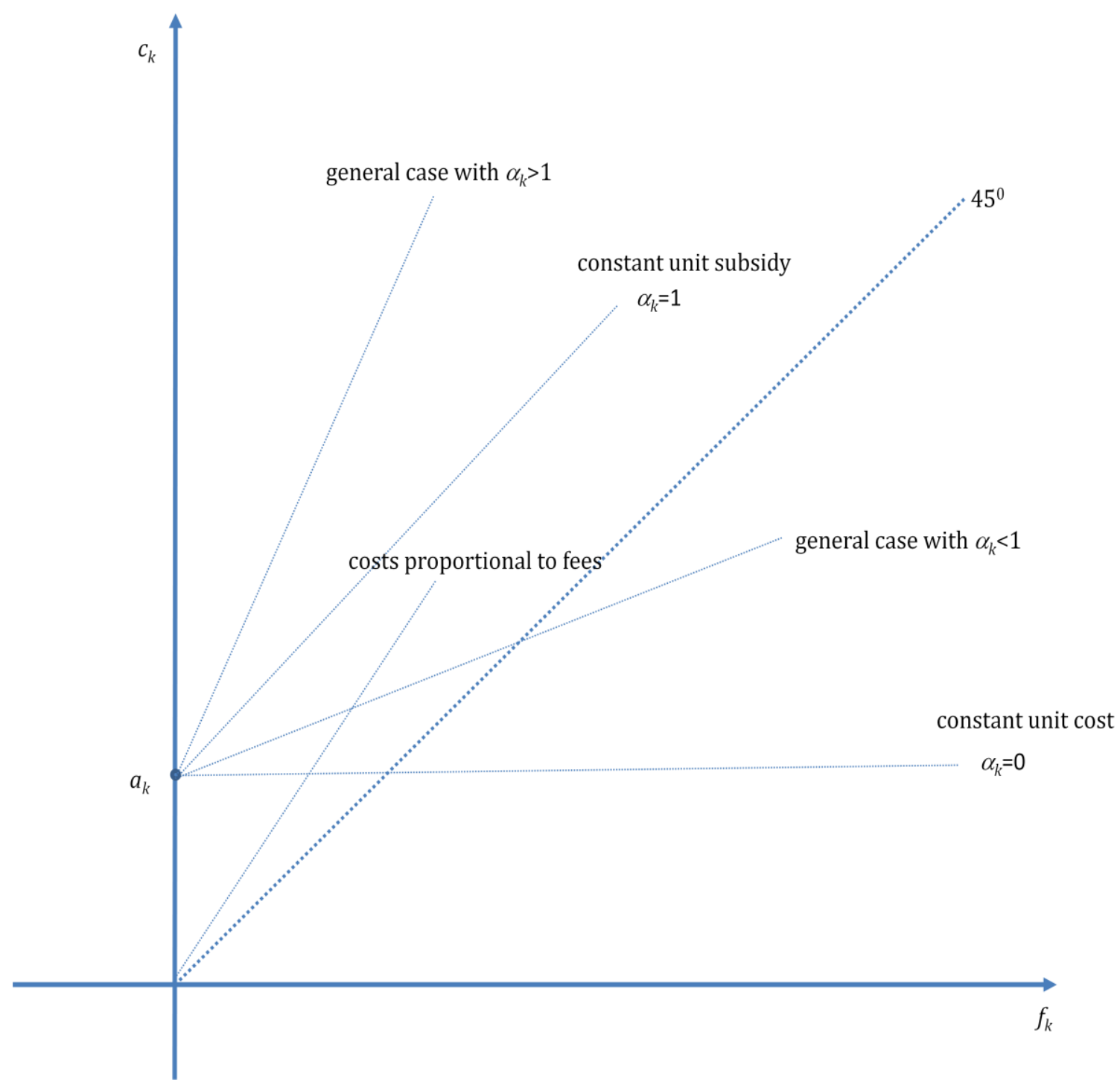


Figure 2: How subsidy concentration index depends on assumptions about link between fees and costs

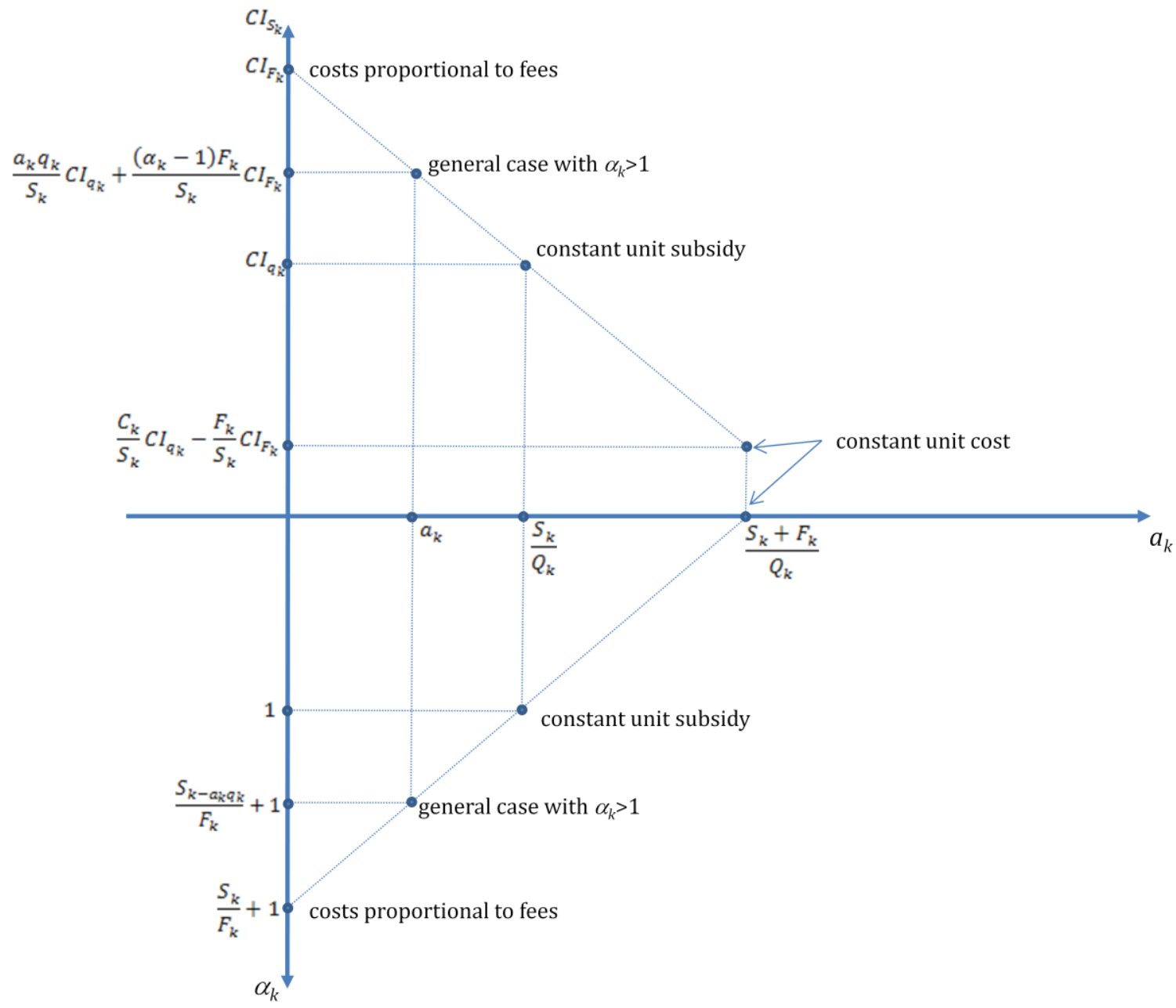


Table 1: Summary of theoretical results

\begin{tabular}{llll}
\hline & $a_{k}$ & $\alpha_{k}$ & $C I_{S_{k}}$ \\
\hline Constant unit cost assumption & $c_{k}=\left(S_{k}+F_{k}\right) / Q_{k}$ & 0 & $\frac{C_{k}}{S_{k}} C I_{q_{k}}-\frac{F_{k}}{S_{k}} C I_{F_{k}}$ \\
Constant unit subsidy assumption & $a_{k}=S_{k} / Q_{k}$ & 1 & $C I_{q_{k}}$ \\
Proportionality assumption & 0 & $\left(S_{k} / F_{k}\right)+1$ & $C I_{F_{k}}$ \\
General assumption & $a_{k}$ & $\alpha_{k}$ & $\frac{a_{k} q_{k}}{S_{k}} C I_{q_{k}}+\frac{\left(\alpha_{k}-1\right) F_{k}}{S_{k}} C I_{F_{k}}$ \\
\hline
\end{tabular}


Table 2: Utilization and out-of-pocket spending, by subsector, based on VHLSS data ${ }^{5}$

\begin{tabular}{lccc}
\hline \multicolumn{1}{c}{ Variable } & $\begin{array}{c}\text { Mean per } \\
\text { person }\end{array}$ & $\begin{array}{c}\text { Aggregate } \\
\text { figure for } \\
\text { Vietnam }\end{array}$ & $\begin{array}{c}\text { Concentration } \\
\text { index }(C l)\end{array}$ \\
\hline Visits/admissions per person p.a. $\left(q_{k}\right)$ & & & \\
Outpatient: CHC & 0.30 & $24,934,564$ & -0.1167 \\
Outpatient: polyclinic & 0.04 & $3,360,418$ & 0.1153 \\
Outpatient: hospital & 0.38 & $31,737,412$ & 0.3050 \\
Inpatient: hospital & 0.07 & $6,124,170$ & 0.1351 \\
& & & \\
Out-of-pocket spending per person p.a. $\left(F_{k}\right)$ & & & \\
Outpatient: CHC & 7.25 & 598,103 & -0.0306 \\
Outpatient: polyclinic & 2.85 & 235,070 & 0.4796 \\
Outpatient: hospital & 71.34 & $5,884,149$ & 0.5174 \\
Inpatient: hospital & 106.96 & $8,822,251$ & 0.4610 \\
\hline
\end{tabular}

Table 3: Aggregate NHA data for $\mathrm{BIA}^{6}$

\begin{tabular}{lcccc}
\hline & & Outpatient & & Inpatient \\
& CHC & Polyclinic & Hospital & Hospital \\
\hline Govt. spending $(S)$ & 162,481 & 21,898 & $3,971,381$ & $3,276,459$ \\
Out-of-pocket spending $(F)$ & 526,035 & 70,894 & $4,919,599$ & $1,729,406$ \\
\hline
\end{tabular}


Table 4: Inequalities in subsidies according to different assumptions ${ }^{7}$

\begin{tabular}{|c|c|c|c|c|c|}
\hline & \multicolumn{3}{|c|}{ Outpatient } & \multirow{2}{*}{$\begin{array}{l}\text { Inpatient } \\
\text { Hospital }\end{array}$} & \multirow[b]{2}{*}{ Total } \\
\hline & $\mathrm{CHC}$ & Polyclinic & Hospital & & \\
\hline \multicolumn{6}{|l|}{$\begin{array}{l}\text { a) Constant unit cost assumption } \\
\text { i) Grossed-up survey data }\end{array}$} \\
\hline$a$ & 30,503 & 76,469 & 310,534 & $1,975,567$ & \\
\hline$\alpha$ & 0 & 0 & 0 & 0 & \\
\hline \multicolumn{6}{|l|}{ Concentration indices } \\
\hline Negative subsidies not set to zero & -0.4335 & -3.7947 & -0.0096 & -0.7423 & -0.3530 \\
\hline Negative subsidies set to zero & -0.1407 & 0.0343 & 0.2471 & 0.0222 & 0.1389 \\
\hline \multicolumn{6}{|l|}{ ii) NHA data } \\
\hline$a$ & 27,613 & 27,613 & 280,142 & 817,395 & \\
\hline$\alpha$ & 0 & 0 & 0 & 0 & \\
\hline \multicolumn{6}{|l|}{ Concentration indices } \\
\hline Negative subsidies not set to zero & -0.3953 & -1.0639 & 0.0420 & -0.0369 & -0.0056 \\
\hline Negative subsidies set to zero & -0.1417 & 0.0633 & 0.2448 & -0.0117 & 0.1228 \\
\hline \multicolumn{6}{|l|}{ b) Constant unit subsidy assumption } \\
\hline$a$ & 6,516 & 6,516 & 125,133 & 535,004 & \\
\hline$\alpha$ & 1 & 1 & 1 & 1 & \\
\hline Concentration indices & -0.1167 & 0.1153 & 0.3050 & 0.1351 & 0.2204 \\
\hline \multicolumn{6}{|l|}{ c) General assumption (intermediate) } \\
\hline$a$ & 6,516 & 6,516 & 36,920 & 184,600 & \\
\hline$\alpha$ & 1.0000 & 1.0000 & 1.4758 & 1.2432 & \\
\hline Concentration indices & -0.1167 & 0.1153 & 0.4547 & 0.3485 & 0.3944 \\
\hline \multicolumn{6}{|l|}{ d) Proportionality assumption } \\
\hline$a$ & 0 & 0 & 0 & 0 & \\
\hline$\alpha$ & 1.2717 & 1.0932 & 1.6749 & 1.3714 & \\
\hline Concentration indices & -0.0306 & 0.4796 & 0.5174 & 0.4610 & 0.4804 \\
\hline
\end{tabular}


Table 5: Inequalities in subsidies factoring in insurance ${ }^{8}$

\begin{tabular}{|c|c|c|c|c|c|}
\hline & \multicolumn{3}{|c|}{ Outpatient } & \multirow{2}{*}{$\begin{array}{l}\text { Inpatient } \\
\text { Hospital }\end{array}$} & \multirow[b]{2}{*}{ Total } \\
\hline & $\mathrm{CHC}$ & Polyclinic & Hospital & & \\
\hline \multicolumn{6}{|c|}{ b) Constant unit subsidy assumption } \\
\hline$a$ & 6,516 & 6,516 & 110,748 & 429,773 & \\
\hline$\alpha$ & 1 & 1 & 1 & 1 & \\
\hline Concentration indices & -0.1167 & 0.1153 & 0.3445 & 0.2288 & 0.2814 \\
\hline \multicolumn{6}{|c|}{ d) Proportionality assumption } \\
\hline$a$ & 0 & 0 & 0 & 0 & \\
\hline$\alpha$ & 1.2717 & 1.0932 & 1.3384 & 1.1473 & \\
\hline Concentration indices & -0.0306 & 0.4796 & 0.5286 & 0.4464 & 0.4787 \\
\hline
\end{tabular}




\section{References}

Castro-Leal, F., J. Dayton, L. Demery and K. Mehra (1999). "Public social spending in Africa: Do the poor benefit?" World Bank Research Observer 14(1): 49-72.

Filmer, D. (2003). The Incidence of Public Expenditures on Health and Education. Washington,D.C., World Bank. Background note for the World Development Report 2004: Making Services Work for the Poor.

Hammer, J., I. BNabi and J. Cercone (1995). Distributional effects of social sector expenditures in Malaysia 1974-89. In: Public Spending and the Poor: Theory and Evidence. D. van de Walle and K. Nead (ed). Baltimore MD, Johns Hopkins University Press.

Kakwani, N., A. Wagstaff and E. van Doorslaer (1997). "Socioeconomic Inequalities in Health: Measurement, Computation, and Statistical Inference." Journal of Econometrics 77 1: 87-103.

Knowles, J., T. Nguyen, B. Dang, K. Nguyen, T. Tran, K. Nguyen and N. Vu (2005). Making Health Care More Affordable for the Poor: Health Financing in Vietnam. Hanoi, Medical Publishing House.

Lieberman, S. S. and A. Wagstaff (2009). Health Financing and Delivery in Vietnam: Looking Forward. Washington DC, World Bank.

O'Donnell, O., E. van Doorslaer, R. P. Rannan-Eliya, A. Somanathan, S. R. Adhikari, D. Harbianto, C. G. Garg, P. Hanvoravongchai, M. N. Huq, A. Karan, G. M. Leung, C.-w. Ng, B. R. Pande, K. Tin, L. Trisnantoro, C. Vasavid, Y. Zhang and Y. Zhao (2007). "The incidence of public spending on healthcare: Comparative evidence from Asia." World Bank Economic Review 21 1: 93-123.

O'Donnell, O., E. van Doorslaer, A. Wagstaff and M. Lindelow (2008). Analyzing Health Equity Using Household Survey Data: A Guide to Techniques and Their Implementation. Washington DC, World Bank.

Prescott, N. (1997). Poverty, social services and safety nets in Vietnam. Washington DC, World Bank. World Bank Discussion Paper No. 376.

Sahn, D. and S. Younger (2000). "Expenditure incidence in Africa: microeconomic evidence." Fiscal Studies 21(3): 329-348.

van de Walle, D. (1995). The distribution of subsidies through public health services in Indonesia. In: Public spending and the poor: Theory and evidence. D. van de Walle and K. Nead (ed). Baltimore, Johns Hopkins University Press.

Wagstaff, A. (2005). "Inequality decomposition and geographic targeting with applications to China and Vietnam." Health Economics 14(6): 649-653.

Wagstaff, A. (2010). "Estimating Health Insurance Impacts under Unobserved Heterogeneity: The Case of Vietnam's Health Care Fund for the Poor." Health Economics 19(2): 189-208. 


\section{Footnotes}

${ }^{1}$ Hammer et al. compute the unit subsidy for each provider type by subtracting the unit fee from the unit cost (or government expenditure).

${ }^{2}$ The data can be downloaded from http://www.who.int/nha/country/vnm/en/.

${ }^{3}$ One problem for the second approach above is that informal payments may not be levied according to the fee schedule, so assuming subsidies are proportional to fees recorded in the survey may be problematic. This is not a reason to scale down user fees in the survey, though, or to favor the traditional approach to BIA. Rather it would be a reason to try to capture explicitly informal payments and to treat them separately - perhaps assuming they are proportional to income rather than linked to the fee schedule.

${ }^{4}$ Note that in this case the concentration index for polyclinic visits is outside the 'normal' interval for a concentration index, i.e. the range when the values of the underlying variable are all nonnegative.

${ }^{5}$ The survey includes 39,071 cases, each with an average sampling weight of 82,480,853. Spending figures are in thousands of Vietnamese Dong. CHC stands for 'commune health center'. The means and the concentration indices are computed from the 2006 VHLSS; sampling weights are used in their computation. The concentration index is computed by ranking individuals according to their per capita household consumption, inclusive of food consumption (whether domestically produced or purchased out of the home), but exclusive of health expenditures.

${ }^{6}$ The government spending (i.e. subsidies) and out-of-pocket payments to public facilities (i.e. fees) are in millions of VND are relate to 2005 , the closest year to 2006 for which detailed NHA data are available. The data are taken from the NHA Table 2. The government spending figures reflect spending by health ministry and central level, and health departments at the provincial, and district levels, as well as spending by the social health insurance agency, Vietnam Social Security. Out-of-pocket payments are listed as spending by households in the NHA Table 2. The table does not break government and out-of-pocket spending down exactly by provider type. The assumption is that spending on 'traditional medicine' is all at the hospital level. Government and out-of-pocket spending on inpatient care are taken from the figures labeled 'inpatient treatment' and 'outpatient treatment' under the 'traditional medicine' heading, i.e. items HD 1.1.1 and HD 1.1.2 respectively. Spending at CHC and polyclinic level are taken from the heading 'primary health care and school health care' under 'health prevention and public health', i.e. item HD 1.2.4. This is the only item in the NHA where spending at the commune level is recorded, but it is possible that some of the polyclinic spending is recorded at a higher level. It was assumed that $75 \%$ of the 'primary health care and school health care' was incurred in health facilities, and the rest in schools.

${ }^{7}$ Unit costs in the constant-cost case are calculated by dividing the sum of subsidies $(S)$ and fees $(F)$ by utilization $(q)$, as explained in the text. Where negative subsidies are set to zero, the subsidies are computed at the individual level in the data, negative values are set to zero, and the concentration index for subsidies is computed from the resulting truncated distribution. The remaining concentration indices for subsidies are computed using the concentration indices in Table 2 and the appropriate expressions in the text, i.e. eqn (2) in the case of the constant-cost assumption, eqn (3) in the case of the proportionality assumption, and eqn (5) in the general linear case. In the general case, the value of $a$ is chosen as high as possible subject to $\alpha$ not being less than 1 .

${ }^{8}$ The value of $\alpha_{k}$ in the proportional case is obtained by solving eqn (9) inserting $S_{k}$ from the NHA, the aggregate fees for the insured and uninsured from the grossed-up NHA data, a value of a of zero, and values of $\varphi$ equal to $67 \%$ and $72 \%$ for hospital outpatient and inpatient services respectively (no reimbursements were made at the time to providers by the insurer for $\mathrm{CHC}$ and polyclinic care). The concentration indices were computed from the microdata after constructing an imputed subsidy variable using eqn (8). 